\title{
Super Mario Seriality: Nintendo's Narratives and Audience Targeting within the Video Game Console Industry
}

\section{Anthony N. Smith}

At the conclusion of Super Mario Bros. (1986), the archetypal side-scrolling platform game, the player-character Mario confronts his arch-nemesis Bowser for the first time. The demonic monster Bowser - King of the Koopa - awaits Mario upon a drawbridge spanning a lava sea. The player's game-long narrative goal is Mario's freeing of Princess Peach by defeating Bowser, her captor; ${ }^{1}$ the player must guide Mario beneath the Koopa King, who hops up and down hurling axes, having him then leap upon a larger glowing axe hovering at the opposite end of the drawbridge. Successful completion of this task results in the disintegration of the drawbridge and Bowser's descent into the lava below upon which Mario enters an adjacent room where Peach awaits. Screen text conveys her highness' gratitude - 'Thank you Mario!', confirming that the hero's 'quest is over'.

The Kyoto-based company Nintendo developed Super Mario Bros. for its first home video game console, the Nintendo Family Computer, released in Japan in 1983 and rebranded and launched in the West as the Nintendo Entertainment System (NES) in 1985. The company has developed further Super Mario games on each of its subsequent home and handheld video game hardware platforms. One of many such games is New Super Mario Bros. Wii (2009), developed for the Nintendo Wii (the company's fifth generation home console, which launched worldwide in 2006). The game not only reprises the two-dimensional side-scrolling platform-game formula of Super Mario Bros., like many other Super Mario games, it also appropriates the 
specific narrative goal of the original game, Mario's rescue of Peach. Additionally, New Super Mario Bros. Wii closely replicates many more specific narrative details from the original game, including its final showdown scenario between Mario and Bowser. Following the template established by the original game, the Koopa King awaits the player character at the far side of a lava-spanning bridge; again, the player must guide Mario beneath the bounding Bowser, dodging the latter's deadly projectiles (this time, fireballs), and have Mario jump upon a large button (which has replaced the glowing axe) that collapses the bridge, sending Bowser hurtling below.

But on this occasion the quest isn't over. The awaiting princess is revealed as an imposter, not Peach but rather a Magikoopa - a sorcerer servant of Bowser adorned in blonde wig and the princess's trademark pink dress. The Magikoopa sprinkles mystical dust over the lava into which its master fell, causing Bowser - now at least twenty times his previous size - to rise from the fire. To free the genuine Peach, the player must navigate Mario across a set of moving platforms, with a marauding Bowser in hot pursuit, towards a second button, which - once pressed collapses the lava sea floor beneath Bowser's feet.

New Super Mario Bros. Wii's replication and variation of narrative content from the initial entry in the Super Mario series of games dovetails neatly with Nintendo's broad industrial goals in recent years, specifically its audience-targeting strategies. As I go on to detail, the company has, from the mid-2000s onwards, aimed to attract a wide audience of both children and adults new to console gaming, while simultaneously appealing to dedicated video game consumers (so called "hardcore" gamers). ${ }^{2}$ New Super Mario Bros. Wii's reprisal of the basic narrative formula from the original Super Mario Bros. is appropriate for new gamers, as it offers a discrete and coherent narrative experience (Bowser kidnaps Peach, Mario rescues her). Yet 
New Super Mario Bros. Wii's playful variations on narrative elements previously established within the series, such as its reworking of the original Mario-Bowser showdown, has the potential to surprise and delight dedicated players familiar with prior Super Mario games. ${ }^{3}$ This chapter explores further the connections between Nintendo's video game narratives and audience targeting aims. It details in particular how a specific mode of serial storytelling, emerging from Nintendo's engagement with its back catalogue of games and ongoing innovation in video game technologies, serves to target these two distinct audience segments.

This chapter contributes to the games studies literature concerning the unique ways in which video games convey narratives, which I define as storyworlds - that is, spatio-temporal models of story that incorporate characters, props, actions and settings - and their presentations. ${ }^{4}$ As such studies make clear, video games are, at the level of textual artefact, not narrative objects per se, but rather interactive systems that facilitate the emergence of fictional narrative through the playing of games; controlling characters and props, players instigate actions within settings, and from this process video game storyworlds are conveyed via screens. ${ }^{5}$ Although important, this work typically neglects the industrial circumstances that inform video game narratives. These studies therefore usefully articulate how fictional narratives emerge from video games, but fail to account for interplay of creativity, industry and technology that contribute to their formations. Taking an 'historical poetics' approach that links storytelling strategies to their conditions of production and circulation, this chapter accounts for the ways in which a significant industrial practice - namely, audience targeting - can inform narrative ${ }^{6}$ Combining evidence of production - in the form of insights from Nintendo personnel - with narratalogical analyses of the company's games, the chapter explores the ways in which Nintendo narratives are 
configured to meet the requirements of both new and experienced gamers. To contextualise this case study, the chapter first establishes the specific industrial conditions for the recent development of Nintendo's software.

\section{Nintendo narrative contexts}

Nintendo operates within the video game console market, a specific sector of the video game industry concerned with the production, distribution and consumption of games intended for the home and portable console hardware devices currently manufactured by the oligopoly of Sony, Microsoft and Nintendo. The institutions that typically drive video game development in this sector can be divided into three distinct categories: the development studios (that create video games in code form), publishers (that often fund development studios, as well as manufacture, market and distribute the hard copies of video games) and the console hardware manufacturers (that build, market and distribute video game platforms). Publishers and development studios most typically operate separately from hardware manufacturers, releasing “third-party" games for hardware manufacturers' platforms (and paying licence fees to the hardware manufacturers on the basis of game sales). But it is, in addition, common practice for a given hardware manufacturer to seek market differentiation by developing and publishing its own "first party" games exclusively for its own platforms. ${ }^{7}$ Understanding the contexts of Nintendo's video game narratives, requires understanding the company's wider goals and strategies for the hardware for which it designs its games.

In the mid-2000s, Nintendo's wanted to regain the home-console hardware market share it had conceded to Sony and Microsoft over the previous years. Nintendo had been, with the NES and its successor - the Super Nintendo 
Entertainment System (SNES), dominant within this market in the late mid-to-late 1980s and early 1990s (over its chief rival Sega). ${ }^{8}$ But competition from Sony (which entered this market in 1994 with the PlayStation) and Microsoft (whose first console, the Xbox, was released in 2001) led to a decline in Nintendo's share. ${ }^{9}$ By the early 2000s, Nintendo had descended into third place in terms of home console market share (behind Sony and Microsoft) due to the poor sales of its fourth home hardware device, the Nintendo GameCube (released in 2001). ${ }^{10}$

Nintendo responded to the competition by reconfiguring its audience-targeting strategies for both its hardware and software. Part of Sony and Microsoft's success had been built on targeting teenage and adult gamers with "mature" narrative content, such as the third party series Grand Theft Auto, with its violence and lawlessness, and Microsoft's first-party series Halo, a militaristic science fiction saga. As Nintendo company president Satoru Iwata admitted in 2002, there was a widespread perception that Nintendo and its content was heavily skewed towards a pre-teen demographic in comparison to its rivals. ${ }^{11}$ The company needed to change this perception in order to increase the consumption of its hardware (and thus software) by appealing more successfully to a teenage and adult audience. ${ }^{12}$ But rather than focusing exclusively on those "hardcore" consumers who had gravitated towards rival hardware, Nintendo instead prioritised a potentially far wider audience of adult gamers who might not purchase consoles because of their associations with young men and/or typically high levels of game difficulty. ${ }^{13}$ This broad target group included female gamers and older players, two demographics that console manufacturers and console game publishers have often neglected to address. ${ }^{14}$

To this end Nintendo conceived of two new hardware systems - the DS handheld console (released in 2004), and the Wii home console (released in 2006) - 
that would enable the company to distinguish itself from its competitors and simultaneously appeal to non-traditional gamers. The contrast in console user inputs between Nintendo and its competitors most obviously illustrates this point. User inputs have generally become progressively more complex and prohibitive since the days of the NES, with Sony and Microsoft console controllers each incorporating two thumb sticks and a myriad of buttons. But the DS and Wii each possess a highly intuitive and accessible mode of input; the former via a touch screen, the latter via motion control. $^{15}$

Just as the Wii and DS systems were configured for a target audience of nontraditional gamers, so too were many of the first party games that Nintendo developed for the system. Wii Fit (2007), which requires the player to carry out light physical activities while standing upon the Wii Balance Board motion-sensor device, is one such title. As Steven E. Jones and George K. Thiruvathukal observe, the game's goal of health improvement (which challenges common perceptions of video games being unhealthy) was specifically conceived for and marketed towards women who might be averse to traditional "hardcore" console games. ${ }^{16}$ But, while developing new titles tailor-made for a non-traditional gamer audience, the company has also maintained its constituency of "hardcore" gamers by consistently producing its more traditional content, chiefly in the form of new instalments for such long-running series as Super Mario and The Legend of Zelda. ${ }^{17}$ As Iwata acknowledged, while Nintendo's 'primary goal' became the 'expansion of the gamer population' through appeals to non-traditional gaming audiences, the company nevertheless remained committed to developing 'the games most enjoyed by our core fans' ${ }^{18}$

Yet, reflecting Nintendo's broader industrial goals from the mid-2000s onward, developers have been motivated to ensure that these more conventional 
games are also accessible to non-traditional gamers. Approaches to user input in the development of Super Mario Galaxy (2007), a three-dimensional platforming game produced for the Wii by Nintendo's in-house development group, the Entertainment \& Analysis Division (EAD), illustrates this. ${ }^{19}$ As the game's director Takao Shimizu notes, the company's aims since the release of the DS and Wii 'to make games that can be enjoyed by anyone, from the age of 5 to 95 ', drove his team to 'make Super Mario Galaxy a game that can be enjoyed by anyone as well' ${ }^{20}$ To this end, EAD opted, for example, to limit the amount of different buttons that the player is required to press so as to have Mario perform actions in the game. ${ }^{21} \mathrm{With}$ the input configuration for the earlier Super Mario Sunshine (2002), which was developed for the GameCube, four distinct Mario actions are mapped on to four different buttons. With the input configuration of Super Mario Galaxy, however, two actions (jump and crouch) are mapped onto two buttons. Yet Super Mario Galaxy players can have Mario perform additional actions via motion control functionality - a slight waggle of the Wii Remote controller, for example, prompts Mario to spin in midair; according to Shimizu, this control scheme ensures that game play is 'more intuitive, even for those people who usually don't play video games'. ${ }^{22}$

This example of Super Mario Galaxy indicates how the development of Nintendo's first-party games in the Wii/DS era reflected the company's targeting of two distinct audience constituencies. Nintendo's development of a three-dimensional platforming game (a genre that the company had helped pioneer with its Super Mario 64 [1996]) is aimed at dedicated gamers but the efforts to reduce the complexity of the control scheme targets a wider audience of players lacking experience with such challenging games. Having established the importance of hardware to Nintendo's 
marketing strategies, the chapter moves on to specifically consider how aspects of the company's fictional narratives serve these audience-targeting objectives.

This case study focuses on two of Nintendo's most famous video game series: Super Mario and The Legend of Zelda, appropriate choices given their richer narrative content relative to many other Nintendo games. The study includes not only Super Mario and Legend of Zelda games developed for DS and Wii, but also those developed for these consoles' successors, the Wii U (launched in 2012) and the 3DS (launched in 2011); each of these latter systems have in many ways been designed to continue Nintendo's objective of addressing "hardcore" players, while also targeting a wider non-traditional gaming audience. ${ }^{23}$ As the following case study details, developers of Super Mario and Legend of Zelda have in recent years consistently relied on a highly specific mode of serial storytelling to strongly engage the former audience segment, while simultaneously not alienating the latter segment.

\section{Seriality in Nintendo Narratives}

Understanding the Nintendo developers' approach to serial storytelling first requires considering more generally how serial texts operate. In the simplest terms, a serial text is a sequence of narratively connected textual instalments, with the distribution of each new instalment usually separated by an interval from the last. The instalments of many serial texts in video games, as well as in film, comics and television, narratively connect on what Robert C. Allen labels the 'syntagmatic axis', meaning that connections form via chains of related events. ${ }^{24}$ A clear example of this type of serial text in video games would be Microsoft's Halo series. For example, at the conclusion of Halo 3 (2007), the game's player character - a cyborg soldier named Master Chief - enters into cryonic sleep within his damaged craft as it drifts in space; at the 
beginning of Halo 4 (2012), Master Chief is awoken from his sleep as enemy alien forces attack his wrecked vessel. These two games thus serially connect via a coherent chronological sequence of related actions within the Halo storyworld. For Microsoft, the implementation of the conventional syntagmatic serial mode within the Halo series enables it to appeal specifically to a committed "hardcore" gaming audience; the series' sprawling narrative, which not only extends across multiple games, but also tie-in novels and comic books, is appropriate for a target market of highly dedicated consumers who will likely play the series' multiple instalments, and also perhaps engage with its transmedia extensions. The opportunity to delve further into Halo's intricate syntagmatic serial narrative - and discover "what happens next" subsequent to previously played instalments - has the potential to increase the attractiveness of each new Halo instalment to consumers within this audience group.

Super Mario or Legend of Zelda games, however, have always typically avoided making explicit serial connections to other instalments within their respective series along the syntagmatic axis. Each Super Mario and Legend of Zelda game, if played from beginning to end, typically introduces and concludes a self-contained dramatic conflict. Most (although, not all) Super Mario games begin with Bowser's kidnapping of Peach and conclude with Mario's rescue of her. Legend of Zelda games, the first of which debuted in Japan in 1986, also often (although, not always) reiterate a similar "damsel in distress" storyline. Many games within the series begin with a magical villain descending upon the peaceful kingdom of Hyrule and kidnapping its princess, Zelda; these games conclude with the player character - the young adventurer, Link - defeating the villain and rescuing Zelda. In Umberto Eco's terms, the narratives of these Nintendo series typically retain an 'iterative' quality; each game represents 'a virtual beginning, ignoring where the preceding event left 
off', presenting a self-contained tale that fails to convey a progression of events beyond itself. ${ }^{25} \mathrm{He}$ observes, 'The very structure of time falls apart [as a consequence of this narrative mode]... that is, the notion of time that ties one episode to another. ${ }^{26}$ Because Nintendo intends that contemporary Super Mario and Legend of Zelda games attract both an audience of dedicated gamers familiar with these series, but also a far wider audience of non-traditional gamers, adoption of the syntagmatic serial mode would be counterproductive; newcomers, perhaps not only unfamiliar with these series but less committed to gaming in general, might be deterred by the requirement to engage with storylines spanning multiple games. Nintendo therefore continues to refrain from use of this mode. But while the mode's absence complements the company's current aim of appealing to a broad audience the tendency of contemporary instalments within these series to establish strong serial connections along what Allen labels the 'paradigmatic' axis permits Nintendo to appeal to dedicated gamers. Via this axis, associations between the instalments of a series form not via a chronology of depicted events but by inferred thematic parallelism. ${ }^{27}$ For example, the Mario-Bowser face-off at the conclusion of New Super Mario Bros. Wii does not link up with its analogous scene in the original Super Mario Bros. along a syntagmatic axis; yet - due to the strong thematic parallels between them - the scenes do serially connect along a paradigmatic axis.

Nintendo developers' heavy implementation of this serial mode within Super Mario and Legend of Zelda games can afford dedicated gamers certain pleasures related to their prior knowledge of these series. By reprising narrative elements from previous instalments, these games are able to pleasantly surprise knowledgeable players by varying and/or elaborating upon these repeated elements (as the Super Mario Bros. Wii sequence does). Critical responses to these games within the video 
game press (which primarily addresses a "hardcore" audience) indeed emphasise this pleasurable potential for players dedicated to these series. IGN's New Super Mario Bros. U (2012, for WII U) review, for example, suggests, 'For those of us who have been adventuring through the Mushroom Kingdom [a Super Mario setting] for decades, this experience is as much about seeing the clever twists on the formula., ${ }^{28}$ Edge's review of The Legend of Zelda: A Link between Worlds (2013, for 3DS), a game which replicates much of the storyworld setting of The Legend of Zelda: A Link to the Past (1991, for SNES), similarly identifies the gratifications to be had from Nintendo's reworking of the familiar: 'What role does well-trodden ground have in a series trading on the thrill of discovery? Well, Nintendo toys with your memories, sticking to [A Link to the Past's] rough shape only to diverge in surprising ways. ${ }^{, 29}$ The implementation of the paradigmatic serial mode enables Nintendo to directly appeal to an audience of dedicated gamers while not confusing and/or antagonising a broader less-dedicated audience; indeed these players are unlikely to be even aware of the operation of the paradigmatic serial mode. ${ }^{30}$

It is important to acknowledge that examples of paradigmatic seriality can also be located to varying degrees in pre-Wii/DS Super Mario and Legend of Zelda games. The rescuing-Peach-from-Bowser narrative goal of the original Super Mario Bros. for example, is itself a variation on an earlier Nintendo game, Donkey Kong (1981), in which Mario must save a damsel from the clutches of this arcade game's eponymous King Kong-esque villain. What Jones and Thiruvathukal identify as 'a general selfconsciousness with game history at Nintendo' has always filtered into the company's games. ${ }^{31}$ But, reflecting its specific audience targeting goals from the Wii and DS era onwards, Nintendo has relied more heavily on the paradigmatic serial mode within the Super Mario and Legend of Zelda series as the primary means with which to address 
dedicated gamers. The heightened use of pastiche concerning prior Super Mario and Legend of Zelda games to be found in Nintendo's recent output reflects the increased prioritisation of this mode; New Super Mario Bros. (2006, for DS) and New Super Mario Bros. Wii's playful yet comprehensive reworking of the series' original game and The Legend of Zelda: A Link Between World's careful and extensive reconstruction of A Link to the Past's storyworld environment are cases in point. Nintendo's marketing materials attest to the increased prioritisation of the paradigmatic mode. For example, while the box art for the GameCube's Super Mario Sunshine emphasises the new storyworld material that the game introduced to the series, the box art for Super Mario 3D Land (2011, for 3DS) by contrast emphasises the storyworld material that the game reprises from prior games. The Sunshine box advertises Mario's new water-spraying backpack and the new Shine Sprite items he must collect, while the $3 D$ Land box organises iconic props and characters strongly associated with 1980s and early-1990s Super Mario games, such as a green pipe, a brown brick, a gold coin, a Goomba (enemy NPC) and a fire flower "power up", around an image of a leaping Mario. For the non-devotee audience that Nintendo hopes to address, the $3 D$ Land box might promise a fun, breezy adventure in a colourful playground. The back of the box emphasises its ease of access: it is 'a pick up and play' game that provides 'help... if you find yourself stuck' in the form of ingame items. But for the dedicated gamers that the company simultaneously targets with the same product, this box essentially operates as an inventory list for vintage Super Mario. The promise of paradigmatic seriality has become a key selling point in Nintendo's address of dedicated gamers, the company's marketing reflecting the increased centrality of the mode to its in-game narrative strategies. 
This chapter has so far broadly outlined the paradigmatic serial mode on which Nintendo has relied in its development of the Super Mario and Legend of Zelda series, explaining how it permits the company to simultaneously address the contrasting needs of two distinct target audiences. It next closely explores the specific narrative techniques of paradigmatic seriality that Nintendo implements, examining how developers utilise a range of storyworld components - props, settings and characters -to forge serial connections along the paradigmatic axes of the Super Mario and Legend of Zelda series.

\section{Techniques of paradigmatic seriality in Nintendo narratives}

The Legend of Zelda: A Link Between Worlds' technique of establishing paradigmatic links to an earlier game via the reiteration of previously presented settings is common within other contemporary Legend of Zelda games as well as contemporary Super Mario games. Recent Super Mario games, for example, will often combine a similar range of environmental level types, including caves, deserts, ghost houses, snowy mountains and volcanic landscapes; developers often reintroduce musical themes previously associated with particular environmental types, reinforcing paradigmatic association. New Super Mario Bros. and New Super Mario Bros. Wii, for example, each scores its cave levels with an updated version of the same Koji Kondo composition that accompanies the cave levels of the original Super Mario Bros.

Developers of contemporary Super Mario and Legend of Zelda games, however, not only revisit familiar environments but also use them in new ways. For example, The Legend of Zelda: Skyward Sword (2011, for Wii), includes a giant live volcano as an integral part of its landscape, a feature that can be found in certain previous Legend of Zelda games. But Skyward Sword uniquely uses its volcano as a 
complement to the 'sprint dash' mechanic (which the game introduces to the series, and which enables Link to sprint) to innovative effect; as EAD's Kenturo Tominaga, who designed the volcano area, recalls, 'I thought if I combined [sprint dash] with a volcano, with its ups and downs, I could make up something fun based around slopes. ${ }^{32}$ For example, whereas, in The Legend of Zelda: Ocarina of Time (1998), Link must negotiate the volcano via a mountain trail, in Skyward Sword, Link can sprint directly up the steep side of the volcano, dodging the boulders that Bokoblins (enemy NPCs) roll towards him. Skyward Sword reprises a familiar landmark but introduces a novel game play element (the stamina dash) that results in a new kind of storyworld action for the series.

Contemporary Super Mario and Legend of Zelda games not only utilise familiar settings as a platform on which to stage new kinds of storyworld activity, they also generate striking contrasts between old and new environments that may please dedicated gamers. For example, while, as noted, the over-world landscape of The Legend of Zelda: A Link between Worlds does generally emulate that of The Legend of Zelda: A Link to the Past, its individual "dungeon" environments, key game play locations throughout the series, have been 'completely redesigned' and bear little relation to those of $A$ Link to the Past. ${ }^{33}$ This redesign contributes to what Tominaga identifies as the feelings of 'newness and familiarity' that Nintendo wishes to evoke. ${ }^{34}$ Super Mario Galaxy provides a further example of this strategy of simultaneously achieving 'newness and familiarity'. As has become customary for the series, the game opens with Mario in the Mushroom Kingdom, home to Princess Peach. But after a fleet of spaceships steals Peach's castle (with Peach inside), Mario is blasted into an entirely new environment, outer space, in which he must negotiate for the first time competing gravitational pulls across a constellation of small planets. 
Contemporary Super Mario and Legend of Zelda games also juxtapose the familiar with the new via the characters and props installed within their settings. With regard to characters, The Legend of Zelda: Skyward Sword, for example, reprises not only the series-defining characters of Link and Zelda, but also friendly non-playing characters (NPCs), such as the Gorons (a rock-form people who first appear in Ocarina of Time), and enemy NPCs such as the Stalfos (skeleton warriors who appear in the original game of the series). A large and diverse cast of new characters debuts next to the familiar ones, including ancient robots, a mole-like people who dwell in the ground, a giant whale-like creature that roams the skies, and an evil harlequinesque demon lord who serves as the narrative's chief antagonist. With regard to props, Super Mario 3D World (2013, for Wii U), for example, includes iconic "power-up" items that date back to the original Super Mario Bros., such as the red mushroom, which makes Mario larger, and the fire flower, which allows the player character to hurl fireballs at his foes. But $3 D$ World also contains innovative "power up" items new to the series, such as the bell, which bestows a cat suit upon Mario, enabling the player character to run up walls, and the double cherry, which permits the player to control multiple Mario clones in one given area at one given time.

Similar to the ways in which these games reprise familiar settings with new forms of game play, they also frequently vary the behaviours of recurring characters and the functions of recurring props. New Super Mario Bros. 2 (2012, for 3DS), for example, includes a version of the Big Boo enemy NPC - a giant spherical ghost that first appears in Super Mario World (1990, for SNES). As in this earlier game, a Big Boo in New Super Mario Bros. 2 will slowly approach Mario when the player character's back is turned, immediately halting - and covering its eyes with its hands - the instant Mario turns to face it. But, unique to New Super Mario Bros. 2, even if 
Mario does face this enemy, the Big Boo still might peek through his hands and nervously sneak forwards. Such variation of recurring characters is primarily intended to engage those dedicated to the series; regarding the Big Boo's new behaviour, for example, EAD's Masaaki Ishikawa, New Super Mario Bros. 2's art director, notes, 'We thought it might be fun because people familiar with Super Mario games so far may be caught off guard. ${ }^{35}$ A similar motivation led to changing the move sets of recurring enemy NPCs in The Legend of Zelda: Twilight Princess (2006, for Wii); as EAD’s Yoshiyuki Oyama, who designed the game's foes observes, 'Several familiar enemies from previous Zelda games make an appearance.... We have... given them slightly different methods of attack, so both people playing for the first time and experienced Zelda fans will be able to enjoy a fresh challenge. ${ }^{36}$

Recurring props are also sometimes reconfigured in ways that might engage dedicated gamers in particular. Super Mario 3D Land, for example, includes the iconic coin block - a yellow cube with a question mark on its side - from the original Super Mario Bros. As in the original game, if Mario jumps beneath the block in Super Mario 3D Land, hitting the object with his head, a gold coin or "power up" item will typically emerge from it. However, in some cases in $3 D$ Land, if the player has Mario repeatedly bash his head against a block, the item will conceal the player character's head, enabling Mario to negotiate the remainder of the level wearing the block while gold coins continuously eject from it.

This chapter has explored a range of Nintendo's techniques of paradigmatic serilaity but has so far neglected the shifting technologies underpinning these narrative practices. Since the video game console industry's inception in the 1970s, console manufacturers adhere to what John Dovey and Helen W. Kennedy label an 'upgrade culture', ensuring that each generation of hardware platforms marks a 
technological advance on the last. ${ }^{37}$ New generations of console hardware often possess increased sound and graphical capacities and/or new forms of user input. Commercial imperatives drive this industrial activity, as manufacturers strongly rely on technological advances as a means to promote their hardware to consumers. The original PlayStation's ability to render polygonal 3D graphics and the Xbox 360's capacity to deliver "high definition" visuals, for example, were both emphasised in the marketing of the new consoles. ${ }^{38}$ Following the GameCube era, however, Nintendo has generally avoided this ongoing "graphical arms race”, instead differentiating its hardware on the basis of alternate innovations, such as the DS' touch screen input, the Wii's motion control and the 3DS' stereoscopic 3D display. As the next section details, these innovations in user input and screen display have factored significantly into the company's techniques of paradigmatic seriality.

\section{Putting technology into Nintendo's paradigmatic seriality}

EAD's Eiji Aonuma, who manages the software group responsible for new Legend of Zelda titles, and who has served as a key creative figure on the series since the late1990s, emphasises the significance of the particular capabilities of a hardware platform to development. He notes that a key objective in the production of Legend of Zelda titles has been developing game play that complements the specific technological affordances of a given console. ${ }^{39}$ As noted, EAD has, with regard to the DS' touchscreen and the Wii's motion control inputs, used these technologies to reduce the controller complexity of game play, thus appealing to new console gamers. But Nintendo has also used new technologies as another means of simultaneously appealing to dedicated gamers by consistently integrating new hardware capabilities into its practice of paradigmatic seriality. This section continues to demonstrate how 
new entries in Super Mario and Legend of Zelda series establish paradigmatic connections through the reprising of storyworld material. But it does so by considering the ways in which the new hardware technologies either permit this storyworld material to function in new ways (via touch screen or motion control input) or be presented in new ways (via 3D stereoscopic display). Nintendo's exploitation of new hardware to provide variations on familiar narrative themes sustains the appeal to dedicated gamers through the paradigmatic serial mode.

Developed for the DS, The Legend of Zelda: The Phantom Hourglass (2007), for example, includes a boomerang within Link's tool set, an item that dates back to the original Legend of Zelda, yet its host platform's touch-screen input allows the prop to function in a new way. In earlier games, Link's boomerang can only follow straight-line trajectories but in The Phantom Hourglass the player can draw a line on the screen to direct the boomerang on a swerving route. Settings are designed to facilitate, and in some circumstances require, this new prop function. For example, within some "dungeons" Link must throw his boomerang around walls so as to hit switches beyond his perspective. EAD's utilisation of the Wii Motion Plus technology in the development of The Legend of Zelda: Skyward Sword demonstrates a similar practice of reconfiguring well-established props in line with the affordances of a new technology. An example is Link's use of bombs; in previous Legend of Zelda games, the player can merely have Link throw or set down the bombs but in Skyward Sword, the player can have Link roll a bomb along the ground via an analogous gesture with the Wii Motion Plus controller. As with the boomerang in The Phantom Hourglass, the Skyward Sword setting encourages the use of the prop's new function and new kinds of storyworld action. In one location, for instance, Link must skilfully roll a bomb across a slender bridge to demolish a rock dam on the other side. 
In the case of Super Mario 3D Land, however, EAD did not prioritise new forms of storyworld action to complement a new platform technology. Utilising the 3DS' stereoscopic 3D display, the studio instead provided new ways to present familiar storyworld action, delivering what the back of the game's box describes as 'Classic Mario action with a modern twist! ${ }^{40}$ Most typically this process involved appropriating particular actions from earlier 2D Super Mario titles, such as a giant ball and chain pendulum swinging towards Mario, as in Super Mario World, or Bowser breathing fireballs as Mario approaches him, as in Super Mario Bros. 3 (1988, for NES). But whereas these earlier games present props such as fireballs and pendulums as moving across a two-dimensional plane, Super Mario 3D Land alters the perspective on these actions to maximise the hardware's affordances. For example, these same objects are often presented as travelling from the rear of a threedimensional polygonal space towards Mario and the player's perspective and then due to the 3D stereoscopic effect - beyond the screen's frame. EAD was highly motivated to demonstrate the hardware's 3D capabilities via such storyworld actions. As the game's director, Koichi Hayashida, observed, 'When it comes to stereoscopic $3 \mathrm{D}$, everyone on the team wants to make stuff shoot out at you. ${ }^{41}$

The game's utilisation of the hardware's distinct affordances for the purposes of visual effect reflects a broader tendency within the industry as developers respond to perpetual hardware technology upgrades. A new hardware cycle's games typically invite players to admire their technical mastery of spectacle relative to prior games. Super Mario 3D Land, and many other games that revel in spectacular imagery, thus operate, suggests Andrew Mactavish, as 'virtuoso performance[s] of technological expertise'. ${ }^{42}$ Yet EAD's pursuit of technologically-enabled spectacle was tied to its wider techniques of paradigmatic seriality, as the studio typically achieved its 
stereoscopic 3D effects via the reworking of storyworld material (particular characters, props and actions) sourced from prior Super Mario games. In line with these wider techniques, and in ways akin to Skyward Sword and The Phantom Hourglass, 3D Land utilises the distinct affordances of its hardware platform as a means to evoke paradigmatic serial connections within its series; in so doing it invites dedicated gamers to gain pleasure from the variations on earlier narrative that it provides. $^{43}$

\section{Conclusion}

In his public pronouncements, Iwata emphasises the significance of the particular preferences and competencies of audience groups to Nintendo's content creation: The final goal of a product is to resonate with and be accepted by people. You can't just force your way through. By saying 'the point is to be accepted', I mean, if you go to a customer with your idea and you realize they don't understand it, it's more important that they do and you should shift your idea. ... Nintendo developers are extremely insatiable when it comes to whether what they make resonates with customers or not. ${ }^{44}$

This chapter's focus on narrative illustrates some of the ways that Nintendo's prioritisation of audience requirements within development factors into its video games. It shows how Nintendo was able to simultaneously address the contrasting requirements of two distinct audience groups via its reliance on the paradigmatic serial mode. The company consistently appealed to dedicated players via the reprising and reformulating of Super Mario and Legend of Zelda storyworld materials, while ensuring that these narrative practices would not dissuade the wider audiences it wanted to target with the same content. 
By adopting a historical poetics approach, this chapter demonstrates the contingency of video game narrative elements upon production conditions. Yet the current Nintendo context should not be considered representative of the console market or the wider video game industry more generally; instead it should be regarded as one distinct set of production conditions among many within a heterogeneous global marketplace. Further work is therefore needed to map the connections between the medium's diversity of industrial contexts and the content it generates.

\footnotetext{
Notes

${ }^{1}$ This overriding narrative goal is not made explicit within the game itself, but rather by its accompanying instruction manual.

${ }^{2}$ For further understanding concerning the term "hardcore" within a video game context, see Jesper Juul, A Casual Revolution: Reinventing Video Games and their Players (Cambridge: The MIT Press, 2010), 8-10.

${ }^{3}$ On the pleasures to be gained from the combination of repetition of and variation on narrative within a series, see Umberto Eco, The Limits of Interpretation (Bloomington: Indiana University Press, 1990), 91-93. For more on patterns of variation and repetition in narrative, see Omar Calabrese, Neo Baroque: A Sign of the Times, trans. Charles Lambert (Princeton: Princeton University Press, 1992), 27-46; Angela Ndalianis, Neo Baroque Aesthetics in Contemporary Entertainment (Cambridge: The MIT Press, 2004), 71-107.

${ }^{4}$ For more on the term and concept of 'storyworlds', see David Herman, Story Logic: Problems and Possibilities of Narrative (Lincoln: University of Nebraska Press, 2002), 13-14.

${ }^{5}$ Henry Jenkins, 'Game Design as Narrative Architecture', in First Person: New Media as Story, Performance and Game, eds Noah Wardrip-Fruin and Pat Harrigan (Cambridge: MIT, 2004), 118-130; Jesper Juul, Half Real: Video Games between Real Rules and Fictional Worlds (Cambridge: The MIT Press, 2005); Marie-Laure Ryan, Avatars of Story (Minneapolis: University of Minnesota Press, 2006), 181-203; Gordon Calleja, In-Game: From Immersion to Incorporation (Cambridge: The MIT Press, 2011), 113-133; Astrid Ensslin, The Language of Gaming (Basingstoke: Palgrave Macmillan, 2012), $142-157$.

${ }^{6}$ On use of the historical poetics approach in film and television, see David Bordwell, 'Historical Poetics of Cinema', in The Cinematic Text: Methods and Approaches, ed. R. Barton Palmer (New York: AMS Press, 1989), 369-398; Jason Mittell, Complex Television: The Poetics of Contemporary Television Storytelling (New York: NYU Press, forthcoming).

${ }^{7}$ For more on the industrial organisation of the console market, see Jon Dovey and Helen W. Kennedy, Game Cultures: Computer Games as New Media (Maidenhead: Open University Press, 2006), 43-62.

${ }^{8}$ Dominic Arsenault, 'Company Profile: Nintendo', The Video Game Explosion: A History from Pong to PlayStation and Beyond, ed. Mark J. P. Wolf (Westport: Greenwood Press, 2008), 113-114.

${ }^{9}$ Nintendo has, in contrast, sustained its dominance over the handheld video game console market ever since the introduction of its first Game Boy system in 1989. Ibid.

${ }^{10}$ P. J. Huffstutter, 'Nintendo Sees Profit Slump on Weak GameCube Sales', Los Angeles Times, 8 April 2003, http://articles.latimes.com/2003/apr/08/business/fi-nintendo8.

${ }^{11}$ Daniel Sloan, Playing to Wiin: Nintendo and the Video Game Industry's Greatest Comeback (Hoboken: Wiley, 2011), 34-36.

12 Ibid.

${ }^{13}$ Ibid., 101-102.

${ }^{14}$ Steven E. Jones and George K. Thiruvathukal, Codename Revolution: The Nintendo Wii Platform (Cambridge: The MIT Press, 2012), 30-31.
} 
${ }^{15}$ Ibid., 53-77, 118; Juul, A Casual Revolution, 103-128.

${ }^{16}$ Jones and Thiruvathukal, Codename Revolution, 31, 79-80.

${ }^{17}$ The high critical regard for certain contemporary Nintendo games within the dedicated gaming press reflects their continued appeal to "hardcore" audience constituencies. For example, Edge named The Legend of Zelda: Skyward Sword its 'Game of the Year' for 2011, while Eurogamer awarded the same accolade to Super Mario 3D World for 2013. Edge Staff, 'The 2011 Edge Awards: Mainstream', Edge, 6 January 2012, http://www.edge-online.com/features/2011-edge-awards-mainstream/; Martin Robinson, 'Eurogamer's Game of the Year 2013', Eurogamer, 30 December 2013, http://www.eurogamer.net/articles/2013-12-30-eurogamers-game-of-the-year-2013.

${ }^{18}$ Satoru Iwata, 'Iwata Asks. The Legend of Zelda: Twilight Princess. Each Philosophy Benefits from the Existence of the Other', Nintendo.com, date accessed: 25 February 2014, http://iwataasks.nintendo.com/interviews/\#/wii/twilight_princess/0/8.

${ }^{19}$ In a 3D platforming game the action takes place in a three-dimension space, as opposed to on a twodimensional plane.

${ }^{20}$ Satoru Iwata, 'Iwata Asks. Super Mario Galaxy. Volume 1: The Producer and Director. From 5 to 95', Nintendo.com, date accessed: 23 February 2014,

http://iwataasks.nintendo.com/interviews/\#/wii/super_mario_galaxy/0/3.

${ }^{21}$ Satoru Iwata, 'Iwata Asks. Super Mario Galaxy. Volume 1: The Producer and Director. A Mario

Even Beginners Can Play', Nintendo.com, date accessed: 22 February 2014, http://iwataasks.nintendo.com/interviews/\#/wii/super_mario_galaxy/0/2.

${ }^{22}$ Ibid.

${ }^{23}$ President of Nintendo Europe Saturo Shibata, for example, noted that he wanted the handheld 3DS system 'to appeal to long time gamers and newcomers'. Nick Jones, '3DS to appeal to new and old gamers', NowGamer, 19 January 2011,

http://www.nowgamer.com/news/919475/nintendo_3ds_to_appeal_to_new_and_old_gamers.html. ${ }^{24}$ Robert C. Allen, Speaking of Soap Operas (Chapel Hill: University of North Carolina Press, 1985), 69.

${ }^{25}$ Umberto Eco, The Role of the Reader: Explorations in the Semiotic in Texts (London: Hutchinson \& Co., 1981), 117.

${ }^{26}$ Ibid., 113-114. There are exceptions to this rule. The Legend of Zelda: Majora's Mask (2000, for

Nintendo 64) strongly evokes serial connections along a syntagmatic axis, explicitly suggesting that its events follow soon after those of The Legend of Zelda: Ocarina of Time (1998, for Nintendo 64).

Paratextual material furthermore suggests that all Legend of Zelda games connect along the syntagmatic axis to form a single branching timeline (involving multiple storyworld realities), but such syntagmatic connections are rarely explicitly evoked within the games. For more on this timeline, see Patrick Thorpe, ed., The Legend of Zelda: Hyrule Historia, trans. Michael Gombos, Takahiro Moriki, Heidi Plechl, Kumar Sivasubramanian, Aria Tanner and John Thomas (Milwaukie: Dark Horse Books, 2013), 68-136.

${ }^{27}$ Allen, Speaking of Soap Operas, 69-71.

${ }^{28}$ Richard George, 'New Super Mario Bros. U Review', IGN, 15 November 2012, http://uk.ign.com/articles/2012/11/15/new-super-mario-bros-u-review.

${ }^{29}$ Edge Staff, 'The Legend of Zelda: A Link between Worlds Review', Edge, 14 November 2013, http://www.edge-online.com/review/the-legend-of-zelda-a-link-between-worlds-review/.

${ }^{30}$ For discussion of producers (in other media) making efforts to conceal from "casual" audiences the serial narrative connections between the texts of a given series, see Matt Hills, "Absent Epic, Implied Story Arcs, and Variation on a Narrative Theme: Doctor Who (2005-2008) as Cult/Mainstream Television', in Third Person: Authoring and Exploring Vast Narratives, eds Pat Harrigan and Noah Wardrip-Fruin (Cambridge: The MIT Press, 2009), 333-342; Anthony N. Smith, 'Media Contexts of Narrative Design: Dimensions of Specificity within Storytelling Industries' (PhD diss., University of Nottingham, 2013), 195-205.

${ }^{31}$ Jones and Thiruvathukal, Codename Revolution, 18.

${ }^{32}$ Satoru Iwata, 'Iwata Asks. The Legend of Zelda: Skyward Sword. Volume 3. The Dense Volcano and Enemy Monsters', Nintendo.com, date accessed: 19 February 2014, http://iwataasks.nintendo.com/interviews/\#/wii/zelda-skyward-sword/2/0.

${ }^{33}$ Satoru Iwata, 'Iwata Asks. The Legend of Zelda: A Link between Worlds. A Challenge from the Developers', Nintendo.com, date accessed: 26 February 2014, https://iwataasks.nintendo.com/interviews/\#/3ds/a-link-between-worlds/0/6. ${ }^{34}$ Ibid. 


\footnotetext{
${ }^{35}$ Satoru Iwata, 'Iwata Asks. New Super Mario Bros. 2. Cooperation from the Super Mario 3D Land Staff', Nintendo.com, date accessed: 17 February 2014, https://iwataasks.nintendo.com/interviews/\#/3ds/nsmb2/0/1.

${ }^{36}$ Satoru Iwata, 'Iwata Asks. The Legend of Zelda: Twilight Princess. Ideas Born Out of Functionality' Nintendo.com, date accessed: 24 February 2014, http://iwataasks.nintendo.com/interviews/\#/wii/twilight_princess/0/1.

${ }^{37}$ Dovey and Kennedy, Game Cultures, 52.

${ }^{38}$ Stephen Kline, Nick Dyer-Witheford and Greg De Peuter, Digital Play: The Interaction of Technology, Culture and Marketing (Montreal \& Kingston: McGill-Queen's University Press, 2003), 153-154; Juul, A Casual Revolution, 13.

${ }^{39}$ Thorpe, The Legend of Zelda: Hyrule Historia, 328.

${ }^{40}$ Super Mario 3D Land European edition (Nintendo, 2011).

${ }^{41}$ Satoru Iwata, 'Iwata Asks. Volume 1: Super Mario 3D Land. "It’s So High I'm Scared!"”, Nintendo.com, date accessed: 17 February 2014, https://iwataasks.nintendo.com/interviews/\#/3ds/super-mario-3d-land/0/3.

${ }^{42}$ Andrew Mactavish, 'Technological Pleasure: The Performance and Narrative of Technology in HalfLife and other High-Tech Computer Games', in ScreenPlay: Cinema/Videogames/Interfaces, eds Geoff King and Tanya Krzywinska (London: Wallflower, 2002), 34. For more on this topic, see Marie-Laure Ryan, 'Beyond Ludus: Narrative, Videogames and the Split Condition of Digital Textuality', in Videogame, Player, Text, eds Barry Atkins and Tanya Krzywinska (Manchester: Manchester University Press, 2007), 14; Ndalianis, Neo Baroque Aesthetics, 99-104. Geoff King, 'Die Hard/Try Harder: Narrative, Spectacle and Beyond, From Hollywood to Videogame', in ScreenPlay: Cinema/ Videogames/Interfaces, eds Geoff King and Tanya Krzywinska (London: Wallflower, 2002), 57-58. Andrew Darley, Visual Digital Culture: Surface Play and Spectacle in New Media Genres (London: Routledge, 2000), 149-151.

${ }^{43}$ For more on the use of technology in intertextual practices within video games, see Ndalianis, Neo Baroque Aesthetics, 99-104.

${ }^{44}$ Toshi Nakamura, 'Why Nintendo Says it Doesn't Make Art', Kotaku, 1 August 2013, http://kotaku.com/why-nintendo-says-it-doesnt-make-art-986738137.
} 